\title{
Evaluación de la inteligencia emocional del maestro de educación física en centros educativos de la Región de Murcia
}

\author{
Evaluation of emotional intelligence of physical education \\ teacher at schools in the Region of Murcia
}

\author{
María Carboneros Castro¹, Noelia Gutiérrez-Ruiz¹, María Martínez-Quiles ${ }^{1}$ K. Deliautaite ${ }^{2}$ y Salvador Angosto ${ }^{1 *}$ \\ 1 Facultad Ciencias del Deporte. Universidad de Murcia (España). \\ 2 Lithuanian Sport University (Lithuania).
}

\begin{abstract}
Resumen: La inteligencia emocional es un factor psicológico clave para generar un vínculo profesor-alumno y un proceso de enseńanza-aprendizaje óptimo. Por ello, el objetivo del presente estudio fue conocer el nivel de inteligencia emocional en docentes de Educación Física. La muestra constó de 94 maestros de Educación Física de diferentes colegios de la Región murciana. El cuestionario, utilizado de forma electrónica, fue el TMMS24. Los resultados únicamente mostraron una correlación positiva entre la claridad y la reparación emocional, siendo esta última dimensión la que obtuvo mejores puntuaciones entre los participantes. En relación al género, las mujeres manifestaron puntuaciones superiores en la reparación emocional. Por otro lado, los maestros con una experiencia inferior a cinco ańos mostraron valores superiores en inteligencia emocional, mientras que los más expertos obtuvieron valores superiores en reparación emocional. El análisis de estos resultados ayuda a mejorar los procesos de formación de los docentes en este ámbito.

Palabras Clave: Educación Primaria, colegio, docente, educación física, inteligencia emocional
\end{abstract}

Abstract: Emotional intelligence is a key psychological factor to generate a teacher-student bond and an optimal teaching-learning process. Thus, the aim of this study was to know the level of emotional intelligence in Physical Education teachers. The sample consisted of 94 Physical Education teachers from different schools in the Region of Murcia. The questionnaire, used electronically, was the TMMS-24. The results only showed a positive correlation between clarity and emotional repair, with the latter dimension obtaining the highest scores among the participants. In relation to gender, females showed higher scores in emotional repair. On the other hand, teachers with less than five years' experience showed higher values in emotional intelligence, while the more experienced ones obtained higher values in emotional repair. The analysis of these results helps to improve teacher training about emotional intelligence.

Keywords: Primary education, school, teacher, physical education, emotional intelligence.

\section{Introducción}

La inteligencia emocional es un factor fundamental para conseguir un óptimo desarrollo integral del ser humano, haciendo hincapié en el buen desarrollo del ajuste personal, el rendimiento en el trabajo y las relaciones sociales. Goleman (1995) define la inteligencia emocional como "una meta-habilidad que determina el grado de destreza que podemos conseguir en el dominio de nuestras otras facultades" (p.68).

Otros autores, como Mayer y Salovey (1997) explican que la inteligencia emocional se encarga de relacionar diferentes habilidades como la percepción con precisión, valoración y expresión de emociones; la habilidad para acceder y/o generar sentimientos cuando facilitan el pensamiento; también la habilidad para entender la emoción y conocimiento emocional, e incluso para regular emociones que promuevan el crecimiento emocional e intelectual.

En el ámbito educativo, los docentes deben tener un fuerte

Dirección para correspondencia [Correspondence address]: Salvador Angosto Sánchez. Facultad Ciencias del Deporte. Universidad de Murcia. C/ Argentina SN, 30720, Santiago de la Ribera, Murcia (España). E-mail: salvador.a.s@um.es equilibrio emocional para desempeñar su labor eficazmente y conseguir una mayor satisfacción laboral, sin que las situaciones estresantes que pueden vivirse diariamente en un centro escolar les desestabilicen. A su vez, la inteligencia emocional predice el bienestar psicológico y grado de burnout de los docentes (Brotheridge y Grandey, 2002; Casassus, 2007; Millán, García-Álvarez y D’Aubeterre, 2014).

Este control emocional no solo lo realizan a nivel personal, siendo los principales referentes de emociones, sentimiento y actitudes; también es importante que interfieran en el control de emociones de su entorno, como pueden ser los estudiantes, los padres y los otros docentes (Brotheridge y Grandey, 2002; Casassus, 2007; Márquez-Cervantes y Gaeta-González, 2017). Fernández-Berrocal, Extremera y Ramos (2002) resaltan la importancia del equilibrio emocional del maestro y de su papel como "educador emocional" del alumnado.

Los profesores perciben que los alumnos con mejor inteligencia emocional tienen un carácter más positivo, prosocial y menos agresivos e intimidatorio que los demás alumnos, además son evaluados positivamente en el desarrollo de sus competencias (Petrides, Sangareau, Furnham y Frederickson, 
2006). Generalmente el rendimiento escolar de estos alumnos suele ser mayor, obteniendo mejores resultados académicos, mayor asistencia a clase y mejor comportamiento (Qualter, Whiteley, Hutchinson y Pope, 2007), así como mejores hábitos saludables (Costarelli y Stamou, 2009).

Alonso, Gea y Yuste (2013) en concordancia con la docencia y los hábitos saludables, hacen hincapié en la formación emocional de futuros profesores de Educación Física; con la finalidad de conseguir, por parte de alumnado, la experimentación de situaciones motrices que contengan un componente social y afectivo, que permita a su misma vez, la educación en emociones. Para ello los docentes deben priorizar en los alumnos un clima motivacional intrínseco, orientado a la tarea, y no una motivación extrínseca (Gutiérrez, Fontenla, Cons, Rodríguez y Pazos, 2017). Además, los autores Conde y Almagro (2013) explican la importancia del uso de técnicas de enseńanza de indagación, fomentando la resolución pacífica de conflictos y el trabajo el grupo. Las tareas deben tener un componente lúdico que favorezcan la aparición de iniciativa y empatía por parte del alumnado. De este modo, creando entornos favorables de aprendizaje y emociones positivas en el aula se favorece el desarrollo la inteligencia emocional junto al bienestar del alumnado (Fernández-Berrocal y Ruiz-Aranda, 2017).

Atendiendo al contexto deportivo, fuera del ámbito escolar, el bienestar emocional es un factor predominante en las personas que realizan deporte, a nivel competitivo y/o a nivel de ocio (Troncoso, Burgos y López-Walle, 2015; Méndez-Giménez, Fernández-Río, Cecchini-Estrada, 2013). Gutiérrez et al. (2017) defienden que aquellos jóvenes deportistas que presentan una mayor capacidad para comprender y regular sus emociones tienen una probabilidad más elevada de no sentir emociones negativas, como la culpabilidad. Estos niveles altos de inteligencia emocional en los deportistas ayudan tanto a sentir niveles menores de ansiedad y estrés, antes, durante y después de la competición (Lane y Wilson, 2011), como un mayor rendimiento deportivo (Zizzi, Deaner y Hirschhorn, 2003).

Por último, los entrenadores con alto control emocional, promueven y generan climas motivacionales en los que se fomente el conocimiento de las propias emociones, el aprendizaje y desarrollo personal, así como el trabajo colaborativo en sus deportistas (Laborde, Dosseville, Allen, 2016). Por el contrario, aquellos que presentan unos niveles más bajos de inteligencia emocional generan un clima de trabajo más conflictivo (Grijalbo y Guzmán, 2014). Por este motivo, Chacón et al. (2017) defienden el desarrollo de estrategias que ayuden a la comprensión de las propias emociones, que desencadenan en motivaciones autogeneradas asociadas al deporte, con la finalidad de conseguir un mantenimiento de vida activo y saludable.

Para concluir, el objetivo principal de la presente investi- gación es conocer el nivel de inteligencia emocional en docentes de Educación Física de la Región de Murcia, identificando diferencias según el género y los años de experiencia.

\section{Material y método}

\subsection{Muestra}

La muestra estuvo compuesta por un total de 94 maestros especialidad de Educación Física, un 51,1\% hombres y un $48,9 \%$ mujeres, con una edad media de $31,7 \pm 6,1$. La Tabla 1 muestra los estadísticos descriptivos de la muestra. Los maestros obtuvieron la titulación hace 8,6 $\pm 5,9$ años, un 45,6\% eran diplomados y el $54,4 \%$ graduados siendo tres cuartas partes especialistas en Educación Física únicamente. Respecto a la experiencia un $57,6 \%$ ha impartido docencia como interino, siendo los ańos de experiencia media de cuatro ańos.

Tabla 1. Estadístico descriptivos de la muestra.

\begin{tabular}{|c|c|c|}
\hline Variable & M & D.T. \\
\hline Edad media & 31,56 & 6,4 \\
\hline Años de la titulación & 8,85 & 6,0 \\
\hline \multirow[t]{2}{*}{ Años de experiencia } & 4,26 & 5,1 \\
\hline & $\mathrm{N}$ & $\%$ \\
\hline \multicolumn{3}{|l|}{ Género } \\
\hline Hombre & 93 & 58,9 \\
\hline Mujer & 65 & 41,1 \\
\hline \multicolumn{3}{|l|}{ Titulación Magisterio } \\
\hline Diplomado & 45 & 47,9 \\
\hline Graduado & 49 & 52,1 \\
\hline \multicolumn{3}{|l|}{ Especialidad } \\
\hline Educación Física & 68 & 72,3 \\
\hline EF y otra materias & 26 & 27,7 \\
\hline \multicolumn{3}{|c|}{ Experiencia como interino } \\
\hline No & 40 & 42,6 \\
\hline Sí & 54 & 57,4 \\
\hline \multicolumn{3}{|l|}{ Otra titulación } \\
\hline No & 31 & 33,0 \\
\hline Otra carrera & 21 & 22,3 \\
\hline Máster & 40 & 42,6 \\
\hline Doctorado & 2 & 2,1 \\
\hline
\end{tabular}

\subsection{Instrumento}

El instrumento utilizado para el desarrollo de este estudio fue "Trait Meta Mood Scale (TMMS-24)", Versión reducida y adaptada por Fernández-Berrocal, Extremera y Ramos 
(2004) de la original de Salovey, Mayer, Goldman, Turvey y Palfai (1995). Se trata de una escala tipo Likert desde 1 (nada de acuerdo) hasta 5 (totalmente de acuerdo), la cual presenta 24 ítems distribuidos en tres dimensiones: i) atención a los sentimientos (ocho ítems); ii) claridad emocional (ocho ítems); y iii) reparación de emociones (ocho ítems). La fiabilidad de la escala mostró un valor de alfa de Cronbach de 0,891 .

\subsection{Procedimiento}

En primer lugar, se realizó una revisión de la literatura científica existente sobre esta temática. Una vez analizada, se determinó el instrumento del estudio y se realizó vía online a través de la herramienta "Encuestas de la Universidad de Murcia”. Para la selección de la muestra, en primer lugar, se puso en contacto con un centro de preparación de oposiciones para Maestro de Educación Física, con la presentación de una carta resumen de la información del estudio y el instrumento; seguidamente, con el consentimiento del centro de formación, se les facilitó el enlace de la encuesta para que pudieran enviarla a todos sus alumnos opositores de forma telemática, vía correo electrónico, en donde aparecía un enlace de encuesta a complementar de forma voluntaria.

\subsection{Análisis de datos}

El análisis de datos se realizó con el paquete estadístico SPSS v.24.0 con licencia de la Universidad de Murcia, se calcularon estadísticos descriptivos de media y desviación típica para las variables cuantitativas y de frecuencias y porcentajes para las variables cualitativas. También se realizó la correlación de Pearson para ver la relación entre las dimensiones de las escalas, la prueba t de Student para observar diferencias entre grupos. El nivel de significación se estableció para un nivel de $\mathrm{p}$ menor a 0,05 .

\section{Resultados}

La Tabla 2 muestra el análisis correlacional sobre las dimensiones del TMMS-24: Atención emocional, Claridad emocional y Reparación emocional. Las dimensiones que obtuvieron mayor correlación, siendo positiva y de nivel moderado $(\mathrm{r}=, 531)$, fueron la claridad emocional y la reparación emocional; contrariamente, la atención emocional obtuvo relaciones positivas de nivel bajo con las otras dos dimensiones, siendo $\mathrm{r}=0,161$ con la claridad emocional y $\mathrm{r}=0,068$ con la reparación emocional.
Tabla 2. Análisis correlaciones TMMS-24.

\begin{tabular}{lccc}
\hline Variable & 1 & 2 & 3 \\
\hline \multirow{2}{*}{ 1. Atención emocional } & 1 & & \\
$\sum_{-1}^{ \pm}$2. Claridad emocional &, 161 & 1 & \\
\hline
\end{tabular}

Nota: ${ }^{* *} p \leq, 001$.

A continuación, en la tabla 3, se muestran los estadísticos comparativos generales de las distintas dimensiones del estudio. Las dimensiones de reparación emocional $(M=4,04 \pm 0,7)$ y claridad emocional $(M=3,94 \pm 0,7)$ obtuvieron valores similares y cercanos a cuatro puntos. Por otro lado, la dimensión de atención emocional mostró valores más bajos que las ante-

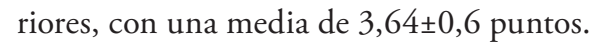

Analizando individualmente cada dimensión, en la atención emocional, los aspectos en los que más incidieron los maestros de educación física, todas ellas con puntuaciones por encima de cuatro puntos, fueron la atención a los sentimientos de los demás $(M=4,43 \pm 0,7)$, prestar atención a su propio estado emocional $(\mathrm{M}=4,30 \pm 0,8)$ y la preocupación por los propios sentimientos $(M=4,28 \pm 0,8)$. Los ítems menos considerados fueron que no piensan a menudo en sus sentimientos, reconocen que muchas veces dejan que los sentimientos afecten a los pensamientos y, la menor valora$\mathrm{da}$, es que no piensan continuamente en su estado de ánimo $(\mathrm{M}=2,74 \pm 1,1)$.

Respecto a los ítems de la claridad emocional, todos estuvieron rondando los cuatro puntos por encima o debajo, siendo las puntuaciones de todos los ítems muy próximas. Los ítems más destacados fueron que llegan a comprender sus propios sentimientos, tienen claros los mismos y suelen ser conscientes de los propios sentimientos en diferentes contextos. Sin embargo, encontraron mayores dificultades en saber decir cómo se sienten a otras personas.

En último lugar, los ítems que conforman la reparación emocional, al igual que la dimensión anterior, tuvieron puntuaciones rondando los cuatro puntos. El aspecto más destacado fue que los maestros cuando están felices tienen mucha energía $(M=4,65 \pm 0,6)$ o se preocupan por tener un buen estado de ánimo $(M=4,11 \pm 0,9)$, o a pesar de no encontrarse bien suelen ser optimistas. Peores puntuaciones aquellos aspectos referidos al control de la propia relajación cuando le dan vueltas a las cosas $(M=3,84 \pm 1,1)$ o si cuando están tristes, piensan en los placeres de la vida $(M=3,66 \pm 1,1)$. 
Tabla 3. Estadísticos descriptivos del TMMS-24.

\begin{tabular}{|c|c|c|}
\hline Ítems & M & D.T. \\
\hline Dimensión Atención emocional & 3,64 & 0,6 \\
\hline 1. Presto mucha atención a los sentimientos. & 4,43 & 0,7 \\
\hline 2. Normalmente me preocupo mucho por lo que siento. & 4,28 & 0,8 \\
\hline 3. Normalmente dedico tiempo a pensar en mis emociones. & 3,83 & 0,9 \\
\hline 4. Pienso que merece la pena prestar atención a mis emociones y estado de ánimo. & 4,30 & 0,8 \\
\hline 5. Dejo que mis sentimientos afecten a mis pensamientos. & 3,12 & 1,1 \\
\hline 6. Pienso en mi estado de ánimo constantemente. & 2,74 & 1,1 \\
\hline 7. A menudo pienso en mis sentimientos. & 3,15 & 1,0 \\
\hline 8. Presto mucha atención a cómo me siento. & 3,29 & 1,1 \\
\hline Dimensión Claridad Emocional & 3,94 & 0,7 \\
\hline 9. Tengo claros mis sentimientos. & 4,06 & 0,8 \\
\hline 10. Frecuentemente puedo definir mis sentimientos. & 3,87 & 0,9 \\
\hline 11. Casi siempre sé cómo me siento. & 3,95 & 1,0 \\
\hline 12. Normalmente conozco mis sentimientos sobre las personas. & 4,01 & 0,8 \\
\hline 13. A menudo me doy cuenta de mis sentimientos en diferentes situaciones. & 4,02 & 0,7 \\
\hline 14. Siempre puedo decir cómo me siento. & 3,67 & 0,9 \\
\hline 15. A veces puedo decir cuáles son mis emociones. & 3,86 & 0,9 \\
\hline 16. Puedo llegar a comprender mis sentimientos. & 4,07 & 0,8 \\
\hline Dimensión Reparación Emocional & 4,04 & 0,7 \\
\hline 17. Aunque a veces me siento triste, suelo tener una visión optimista. & 4,05 & 1,0 \\
\hline 18. Aunque me sienta mal, procuro pensar en cosas agradables. & 4,01 & 0,8 \\
\hline 19. Cuando estoy triste, pienso en todos los placeres de la vida. & 3,66 & 1,1 \\
\hline 20. Intento tener pensamientos positivos aunque me sienta mal. & 3,97 & 0,9 \\
\hline 21. Si doy demasiadas vueltas a las cosas, complicándolas, trato de calmarme. & 3,84 & 1,1 \\
\hline 22. Me preocupo por tener un buen estado de ánimo. & 4,11 & 0,9 \\
\hline 23. Tengo mucha energía cuando me siento feliz. & 4,65 & 0,6 \\
\hline 24. Cuando estoy enfadado intento cambiar mi estado de ánimo. & 4,00 & 0,9 \\
\hline
\end{tabular}

En los resultados de la Tabla 4 se muestran los estadísticos comparativos de las dimensiones según el género. La dimensión que consiguió una mayor puntuación fue la reparación emocional con una media de $4,05 \pm 0,7$ puntos en hombres y 4,02 \pm 0.7 puntos en mujeres, seguida de la claridad emocional con la misma puntuación en ambos géneros $(M=3,94 \pm 0,7)$ y la atención emocional (Hombres $=3,50 \pm 0,7$; Mujeres $=3,79 \pm 0,7)$. Existió diferencias estadísticamente significativas según género en la atención emocional $(\mathrm{p}<, 05)$.

Analizando más profundamente cada dimensión, en primer lugar, según la dimensión de atención emocional, los hombres tuvieron mejores valoraciones en que prestan mucha atención a los sentimientos $(M=4,25 \pm 0,7)$ y que consideran que merece la pena prestar atención a las propias emociones $(M=4,15 \pm 0,9)$. Por el contrario, consideraron que no pensaban constantemente en su estado de ánimo o no pienses constantemente en los propios sentimientos $(M=2,92 \pm 1,0)$. Por su parte, las mujeres, generalmente tuvieron puntuaciones superiores a los hombres en todos los ítems de la atención emocional. Siendo los ítems mejor puntuados los mismos que los hombres, además de que no se preocupan mucho por lo que sienten $(\mathrm{M}=4,37 \pm 0,7)$. El ítem 2 "presto mucha atención a los sentimientos" y el ítem 7 "a menudo pienso en mis sentimientos" mostraron diferencias significativas según género $(\mathrm{p} \leq, 05)$, mientras que el ítem 4 "pienso que merece la pena prestar atención a mis emociones y estado de ánimo” tuvo una alta tendencia a la significación $(\mathrm{p}=, 055)$.

La claridad emocional tuvo diferencias en cuanto a los aspectos que mayor atención prestan los hombres y las mujeres. Los hombres tuvieron mejores puntuaciones en aspectos como tener claros sus sentimientos o llegar a comprenderlos, ambos ítems con una valoración de $4,17 \pm 0,8$ puntos, siendo 
lo menos valorado aspectos como la definición de los propios sentimientos o decir lo que uno siente. Las mujeres, a diferencia de los hombres, tuvieron en mayor consideración aspectos como saber siempre lo que se siente o saber definir sus propios sentimientos con puntuaciones medias de 4,02 $\pm 0,9$ y $4,00 \pm 0,8$ respectivamente. Sin embargo, al igual que los hombres no saben cómo decir lo que sienten ( $M=3,72 \pm 0,9)$.

Generalmente en la dimensión reparación emocional los hombres presentaron puntuaciones más altas que las mujeres en la mayoría de ítems, siendo los mejor valorados por los hombres aquellos aspectos relacionados con tener mucha energía al sentirse feliz $(M=4,52 \pm 0,7)$, preocuparse por tener un buen estado de ánimo, tener visión optimista aunque no se encuentre bien o pensar en cosas agradables en esas situaciones. Todos estos aspectos tuvieron puntuaciones superiores a cuatro puntos. Mientras las mujeres valoraron en mayor medida tener mucha energía cuando están felices $(M=4,78 \pm 0,5)$, la preocupación por tener un buen estado de ánimo o cuando se enfadan intentan cambiar ese estado de ánimo. Los resultados del ítem 23 "tengo mucha energía cuando me siento feliz” mostró diferencias estadísticamente significativas según género $(\mathrm{p} \leq, 05)$.

Tabla 4. Estadísticos comparativos ítems TMMS-24 según género.

\begin{tabular}{|c|c|c|c|c|c|}
\hline \multirow{2}{*}{ Ítems } & \multicolumn{2}{|c|}{ Hombre } & \multicolumn{2}{|c|}{ Mujer } & \multirow{2}{*}{ p valor } \\
\hline & M & D.T. & M & D.T. & \\
\hline Dimensión atención emocional & 3,50 & 0,7 & 3,79 & 0,6 &, $024^{*}$ \\
\hline 1. Presto mucha atención a los sentimientos. & 4,25 & 0,7 & 4,61 & 0,6 &, $008^{*}$ \\
\hline 2. Normalmente me preocupo mucho por lo que siento. & 4,19 & 0,8 & 4,37 & 0,7 &, 261 \\
\hline 3. Normalmente dedico tiempo a pensar en mis emociones. & 3,79 & 0,9 & 3,87 & 0,8 & ,669 \\
\hline 4. Pienso que merece la pena prestar atención a mis emociones y estado de ánimo. & 4,15 & 0,9 & 4,46 & 0,7 & 055 \\
\hline 5. Dejo que mis sentimientos afecten a mis pensamientos. & 2,94 & 1,2 & 3,30 & 1,0 &, 111 \\
\hline 6. Pienso en mi estado de ánimo constantemente. & 2,65 & 1,0 & 2,85 & 1,1 & ,371 \\
\hline 7. A menudo pienso en mis sentimientos. & 2,92 & 1,0 & 3,39 & 1,0 &, $024^{*}$ \\
\hline 8. Presto mucha atención a cómo me siento. & 3,10 & 1,2 & 3,48 & 1,0 &, 100 \\
\hline Dimensión Claridad Emocional & 3,94 & 0,7 & 3,94 & 0,7 & 970 \\
\hline 9. Tengo claros mis sentimientos. & 4,17 & 0,8 & 3,96 & 0,8 &, 213 \\
\hline 10. Frecuentemente puedo definir mis sentimientos. & 3,75 & 1,0 & 4,00 & 0,8 &, 181 \\
\hline 11. Casi siempre sé cómo me siento. & 3,88 & 1,1 & 4,02 & 0,9 &, 474 \\
\hline 12. Normalmente conozco mis sentimientos sobre las personas. & 4,04 & 0,8 & 3,98 & 0,7 & ,686 \\
\hline 13. A menudo me doy cuenta de mis sentimientos en diferentes situaciones. & 4,06 & 0,7 & 3,98 & 0,8 &, 588 \\
\hline 14. Siempre puedo decir cómo me siento. & 3,63 & 0,9 & 3,72 & 0,9 & ,633 \\
\hline 15. A veces puedo decir cuáles son mis emociones. & 3,85 & 0,9 & 3,87 & 0,8 & ,931 \\
\hline 16. Puedo llegar a comprender mis sentimientos. & 4,17 & 0,8 & 3,98 & 0,9 & ,283 \\
\hline Dimensión Reparación emocional & 4,05 & 0,7 & 4,02 & 0,7 & ,869 \\
\hline 17. Aunque a veces me siento triste, suelo tener una visión optimista. & 4,15 & 1,0 & 3,96 & 1,0 & ,356 \\
\hline 18. Aunque me sienta mal, procuro pensar en cosas agradables. & 4,10 & 0,8 & 3,91 & 0,9 & 277 \\
\hline 19. Cuando estoy triste, pienso en todos los placeres de la vida. & 3,73 & 1,0 & 3,59 & 1,1 &, 528 \\
\hline 20. Intento tener pensamientos positivos aunque me sienta mal. & 3,98 & 0,9 & 3,96 & 0,9 & ,906 \\
\hline 21. Si doy demasiadas vueltas a las cosas, complicándolas, trato de calmarme. & 3,85 & 1,1 & 3,83 & 1,2 & ,902 \\
\hline 22. Me preocupo por tener un buen estado de ánimo. & 4,13 & 0,9 & 4,09 & 0,9 &, 845 \\
\hline 23. Tengo mucha energía cuando me siento feliz. & 4,52 & 0,7 & 4,78 & 0,5 &, $045^{*}$ \\
\hline 24. Cuando estoy enfadado intento cambiar mi estado de ánimo. & 3,92 & 0,9 & 4,09 & 0,8 &, 350 \\
\hline
\end{tabular}

Nota: $\mathrm{p} \leq, 05$

Finalmente, en la Tabla 5, se exponen los resultados estadísticos comparativos según la experiencia de los do- centes, con una duración menor o igual o mayor a cinco años. Al igual que los resultados anteriores, la dimensión 
con mayor puntuación en ambos grupos fue la reparación emocional (Menos 5 años=3,98 $\pm 0,7$; Más 5 años=4,14 $\pm 0,5$ ), seguida de la claridad emocional (Menos 5 años=3,93 $\pm 0,7$; Más 5 años $=3,96 \pm 0,7$ ) y la atención emocional (Menos de 5

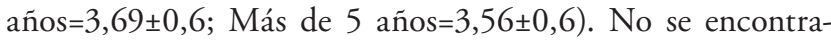
ron diferencias estadísticamente significativas en ninguna dimensión ( $p>, 05)$.

Enfocándose en cada dimensión, ambos grupos tuvieron sus mejores puntuaciones en la atención emocional sobre aspectos como prestar atención a los sentimientos, la preocupación por lo que sienten o piensan que merece la pena prestar atención a las emociones y estado de ánimo, con valores superiores a cuatro puntos. Por el contrario, los aspectos peor valorados fueron ejar que los sentimientos les afecten, pensar en su estado de ánimo continuamente o en sus sentimientos. Estos dos últimos aspectos junto el ítem 8 "presto mucha atención a cómo me siento" presentaron una alta tendencia a la significación según la experiencia docente $(\mathrm{p}<, 10)$.

Los maestros con menor experiencia valoraron mejor en la claridad emocional aspectos como que a menudo son conscientes de sentimientos en diferentes situaciones $(M=4,10 \pm 0,7)$, que pueden llegar a comprender sus senti- mientos y los tienen claros, mientras que los maestros con más experiencia también pueden definir sus propios sentimientos $(M=4,03 \pm 1,0)$. Al contrario, los maestros con menos experiencia presentaron mayores dificultades para expresar sus sentimientos, los maestros de mayor experiencia a veces no saben indicar cuáles son las emociones que sienten.

Finalmente, los maestros con mayor experiencia tuvieron puntuaciones más altas en todos los ítems que los maestros con menor experiencia, excepto en que cuando están tristes, piensan en los placeres de la vida, que tuvo una puntuación ligeramente superior (Menos de 5 años=3,67 $\pm 1,1$; Más de 5 años=3,64 $\pm 1,1)$. Este ítem fue el único que no superó los cuatro puntos en los maestros con mayor experiencia, mientras que los únicos aspectos en los que los maestros menos experimentados tuvieron valores superiores a cuatro puntos fueron en que tienen mucha energía cuando son felices $(\mathrm{M}=4,57 \pm 0,7)$ y se preocupan por tener un buen estado de ánimo $(M=4,08 \pm 1,0)$. Existió diferencias significativas según tiempo de experiencia docente en el ítem 21 "si doy demasiadas vueltas a las cosas, complicándolas, trato de calmarme" $(\mathrm{p}<, 05)$ y una alta tendencia a la significación en el ítem 23 sobre sentirse con energía cuando están felices.

Tabla 5. Estadísticos comparativos ítems TMMS-24 según experiencia.

\begin{tabular}{|c|c|c|c|c|c|}
\hline \multirow{2}{*}{ Ítems } & \multicolumn{2}{|c|}{ Menos de 5 años } & \multicolumn{2}{|c|}{5 años o más } & \multirow{2}{*}{ p valor } \\
\hline & $\mathrm{M}$ & D.T. & $\mathrm{M}$ & D.T. & \\
\hline Dimensión atención emocional & 3,69 &, 6 & 3,56 & 6 & ,347 \\
\hline 1. Presto mucha atención a los sentimientos. & 4,38 & ,7 & 4,52 & ,6 & ,306 \\
\hline 2. Normalmente me preocupo mucho por lo que siento. & 4,26 & 8 & 4,30 & 8 &, 811 \\
\hline 3. Normalmente dedico tiempo a pensar en mis emociones. & 3,75 & ,8 & 3,97 & 1,0 & 257 \\
\hline 4. Pienso que merece la pena prestar atención a mis emociones y estado de ánimo. & 4,28 & ,8 & 4,33 &, 8 &, 750 \\
\hline 5. Dejo que mis sentimientos afecten a mis pensamientos. & 3,20 & 1,1 & 2,97 & 1,1 & ,349 \\
\hline 6. Pienso en mi estado de ánimo constantemente. & 2,90 & 1,1 & 2,45 & 1,1 &, 057 \\
\hline 7. A menudo pienso en mis sentimientos. & 3,30 & 1,0 & 2,88 & 1,1 &, 060 \\
\hline 8. Presto mucha atención a cómo me siento. & 3,43 & 1,1 & 3,03 & 1,0 &, 092 \\
\hline Dimensión Claridad Emocional & 3,93 &, 7 & 3,96 &, 7 & 822 \\
\hline 9. Tengo claros mis sentimientos. & 4,00 & ,8 & 4,18 & ,8 &, 229 \\
\hline 10. Frecuentemente puedo definir mis sentimientos. & 3,79 & 9 & 4,03 & 1,0 &, 870 \\
\hline 11. Casi siempre sé cómo me siento. & 3,93 & 1,0 & 3,97 & 1,0 &, 504 \\
\hline 12. Normalmente conozco mis sentimientos sobre las personas. & 4,05 &, 7 & 3,94 & ,8 & ,175 \\
\hline 13. A menudo me doy cuenta de mis sentimientos en diferentes situaciones. & 4,10 &, 7 & 3,88 & ,9 &, 260 \\
\hline 14. Siempre puedo decir cómo me siento. & 3,59 & 9 & 3,82 & 9 &, 539 \\
\hline 15. A veces puedo decir cuáles son mis emociones. & 3,90 & ,9 & 3,79 & ,9 &, 891 \\
\hline 16. Puedo llegar a comprender mis sentimientos. & 4,07 & ,8 & 4,09 & ,9 & ,239 \\
\hline Dimensión Reparación emocional & 3,98 & ,7 & 4,14 &, 5 & ,204 \\
\hline 17. Aunque a veces me siento triste, suelo tener una visión optimista. & 3,98 & 1,1 & 4,18 & ,8 & ,356 \\
\hline 18. Aunque me sienta mal, procuro pensar en cosas agradables. & 3,95 & ,9 & 4,12 &, 8 & ,356 \\
\hline 19. Cuando estoy triste, pienso en todos los placeres de la vida. & 3,67 & 1,1 & 3,64 & 1,1 &, 880 \\
\hline
\end{tabular}

SPORT TK: Revista Euroamericana de Ciencias del Deporte

ISSN edición web: 2340-8812 / vol. 9, n. 2 (Supl. 1) / Murcia / Noviembre 2020 / Págs. 13-22
Evaluación de la inteligencia emocional del maestro de educación física en... SPORT TK, 9(2) (supl. 1), 13-22 


\begin{tabular}{|c|c|c|c|c|c|}
\hline \multirow{2}{*}{ Ítems } & \multicolumn{2}{|c|}{ Menos de 5 años } & \multicolumn{2}{|c|}{5 años o más } & \multirow{2}{*}{ p valor } \\
\hline & $\mathrm{M}$ & D.T. & $\mathrm{M}$ & D.T. & \\
\hline 20. Intento tener pensamientos positivos aunque me sienta mal. & 3,90 & 1,0 & 4,09 & ,7 & 298 \\
\hline 21. Si doy demasiadas vueltas a las cosas, complicándolas, trato de calmarme. & 3,69 & 1,2 & 4,12 & ,9 &, $046^{*}$ \\
\hline 22. Me preocupo por tener un buen estado de ánimo. & 4,08 & 1,0 & 4,15 & 9 & ,732 \\
\hline 23. Tengo mucha energía cuando me siento feliz. & 4,57 &, 7 & 4,79 &, 5 & 084 \\
\hline 24. Cuando estoy enfadado intento cambiar mi estado de ánimo. & 3,97 & ,9 & 4,06 & ,7 & ,601 \\
\hline
\end{tabular}

Nota: $p \leq, 05$

\section{Discusión}

El objetivo principal de este estudio fue determinar el grado de influencia de la inteligencia emocional en docentes de Educación Física de la Región de Murcia. En primer lugar, las variables que mostraron mayor correlación en el estudio fueron la claridad emocional y la reparación emocional. Igualmente, Extremera y Berrocal (2004) defendieron que estas dos variables eran muy significativas y presentaron resultados favorables en factores positivos de apoyo social, como el compañerismo, el afecto y la autoestima. Además, ambas variables estaban relacionadas con el factor "perspectiva", que recoge la capacidad de adoptar distintas perspectivas ante diversas situaciones; mientras que la variable de atención emocional estaba directamente relacionada con el factor "fantasía", que recoge la capacidad imaginativa del sujeto. Otros autores, como Ferragut y Fierro (2012) también tuvieron una correlación positiva entre las variables de claridad y reparación emocional, y negativa en la atención emocional en la escala de Bienestar Personal (EBP).

En relación al género, la puntuación media fue superior en las mujeres en la variable de atención emocional y en los resultados de las demás variables no se encontraron diferencias significativas. Estos resultados coinciden con Cejudo y López-Delgado (2017), quienes confirmaron en su estudio que las docentes de educación infantil y primaria obtuvieron medias superiores en esta variable. Además, en estas mismas etapas educativas, Sánchez-Huéscar (2018) incluyó mejores resultados por parte de las mujeres, no solo en la variable de atención, sino también en la variable de claridad emocional. Contrariamente, los hombres obtuvieron mejores resultados en la variable de reparación emocional. Sin embargo, otros autores expusieron que las mujeres presentaron medias superiores en las tres dimensiones (Larramendi, 2015; Molero y Pantoja, 2015); contrariamente a otros, que no encontraron diferencias significativas entre las distintas variables (Cazalla-Luna, Ortega-Álvarez y Molero, 2015; Gutiérrez-Moret, Ibáñez-Martínez, Aguilar-Moya y Vidal-Infer, 2016).

Dentro de la variable "atención emocional", el ítem que mostró máxima significación entre géneros fue "presto atención a mis sentimientos". Salovey (2006) explicó que este uso, comprensión y manejo de las emociones es mayor en mujeres que en hombres. De igual modo, las mujeres presentaron diferencias en la inteligencia emocional porque sus rasgos de identidad son más expresivos que los rasgos de lo hombres (Gartzia, Aritzeta, Balluerka, y Heredia, 2012).

Por otro lado, los docentes con una experiencia igual o mayor a cinco años mostraron diferencias significativas en la reparación emocional, respecto a los docentes con menor experiencia. Esto se debe a que los docentes con la experiencia van adquiriendo competencias y construyendo estrategias, para controlar las emociones negativas y fomentar las positivas (Hernández y Palomera, 2004; Palomera, Gil-Olarte y Brackett, 2006). El ítem con mayor significación de esta variable fue "si doy demasiadas vueltas a las cosas, complicándolas, trato de carlmarme"; de este modo, los docentes con menos experiencia presentan menor capacidad para neutralizar sensaciones negativas o estresantes y puede desencadenar en un mayor índice de burnout en estos docentes nóveles (Cejudo y López-Delgado, 2017).

Sin embargo, los docentes con una experiencia inferior a cinco años presentaron datos tendientes a la significación en la atención emocional. En este ámbito, López-Luque (2017) explicaba que los docentes noveles presentaron mejor inteligencia emocional porque la formación de docentes en este campo ha mejorado actualmente. Esta formación inicial en inteligencia emocional es un factor primordial para el desarrollo integral del docente y la consecución de la calidad de enseñanza (Palomera, Fernández-Berrocal, Brackett, 2008). Contrariamente, otros autores como Cejudo y López-Delgado (2017) no encontraron diferencias en los niveles de inteligencia emocional acorde a los ańos de experiencia de los docentes.

Los resultados obtenidos de este estudio no pueden ser generalizados ya que no se realizó ningún tipo de muestreo, seleccionando la muestra por conveniencia. Por tanto, futuros estudios deberían contemplar hacer un muestreo de todos los maestros de educación física con residencia en la Región de Murcia o que ejercen en algún centro escolar de la propia comunidad para observar el verdadero nivel de inteligencia emocional. Igualmente, también podría analizarse la inteligencia emocional junto a otras variables y observar su posible incidencia. 


\section{Conclusiones}

Las principales conclusiones que pueden obtenerse de este estudio son que las dimensiones de inteligencia emocional que presentan mayor correlación son la claridad emocional y la reparación emocional; contrariamente, la atención emocional no se correlaciona con ninguna otra dimensión. Entre estas dimensiones existentes, la que obtiene mayor puntuación entre los docentes participantes es la reparación emocional, seguida de la claridad emocional y, por último, la atención emocional.

En relación al género y el desarrollo de inteligencia emocio- nal, las mujeres manifiestan valores superiores a los hombres en la reparación emocional; en las demás variables, atención y claridad emocional, no se aprecian diferencias significativas. Por otro lado, en relación a los ańos de experiencia de los docentes, los que posee una experiencia igual o superior a 5 años, presentan una reparación emocional mayor a los docentes con menor experiencia; por contra, los docentes con una experiencia inferior a 5 ańos, poseen una atención emocional algo superior a los docentes con mayor experiencia. Finalmente, no se encuentran diferencias significativas, en relación a la experiencia del docente, sobre la claridad emocional.

\section{Referencias}

1. Alonso, J. I., Gea, G. y Yuste, J. L. (2013). Formación emocional y juego en futuros docentes de Educación física. Revista Electrónica de Formación del Profesorado, 16(1), 97-108.

2. Brotheridge, C. M. y Grandey, A. A. (2002). Emotional labor and burnout: Comparing two perspectives of "people work". Journal of Vocational Behavior, 60(1), 17-39.

3. Casassus, J. (2007). La educación del ser emocional. Santiago, Chile: Editorial Cuarto Propio.

4. Cazalla-Luna, N., Ortega-Álvarez, F. y Molero, D. (2015). Autoconcepto e inteligencia emocional de docentes en prácticas. Revista Electrónica de Investigación y Docencia (REID), 14, 151-164.

5. Cejudo, J. y López-Delgado, M. L. (2017). Importancia de la inteligencia emocional en la práctica docente: un estudio con maestros. Psicología Educativa, 23(1), 29-36.

6. Chacón, R., Padial, R., Zurita, F., Castro, M., Valero, G. G. y Ramírez, I. (2017). Clima motivacional e inteligencia emocional en la promoción de hábitos saludables: Una revisión narrativa. EmásF: revista digital de educación física, 49, 108-117.

7. Conde, C. y Almagro, B. J. (2013). Estrategias para desarrollar la inteligencia emocional y la motivación en el alumnado de Educación Física. E-motion. Revista de Educación, Motricidad e Investigación, 1, 212-220.

8. Costarelli, V. y Stamou, D. (2009). Emotional Intelligence, Body Image and Disordered Eating Attitudes in Combat Sport Athletes. Journal of Exercise Science \& Fitness, 7(2), 104-111.

9. Extremera, N. y Fernández-Berrocal, P. (2004). Inteligencia emocional, calidad de las relaciones interpersonales y empatía en estudiantes universitarios. Clínica y salud, 15(2), 117-137.

10. Extremera, N. y Fernández-Berrocal, P. (Marzo de 2001). ¿Es la inteligencia emocional un adecuado predictor del rendimiento académico en estudiantes? Conferencia llevada a cabo en las III Jornadas de Innovación Pedagógica: Inteligencia Emocional. Una brújula para el siglo XXI, Granada, España.

11. Extremera, N., y Fernández-Berrocal, P. (2004). La inteligencia emocional: Métodos de evaluación en el aula. Revista Iberoamericana de Educación, 34(1), 1-12.

12. Fernández- Berrocal, P. y Extremera, N. (2002). La inteligencia emocional como una habilidad esencial en la escuela. Revista Iberoamericana de ducación, 29(1), 1-6.

13. Fernández-Berrocal, P. y Ruiz-Aranda, D. (2017). La inteligencia emocional en la educación. Electronic Journal of Research in Education Psychology, 6(15), 421-436.

14. Fernández-Berrocal, P., Extremera, N. y Ramos, N. (2004). Validity and reliability of the spanish modified versión of the trait meta-mood scale. Psychological Reports, 94, 751-755.

15. Ferragut, M., y Fierro, A. (2012). Inteligencia emocional, bienestar per- sonal y rendimiento académico en preadolescentes. Revista latinoamericana de psicología, 44(3), 95-104.

16. Gartzia, L., Aritzeta, A., Balluerka, N. y Heredia, E. B. (2012). Inteligencia emocional y género: más allá de las diferencias sexuales. Anales de PsicologialAnnals of Psychology, 28(2), 567-575.

17. Goleman, D. (1995). Emotional intelligence. New York, Estados Unidos: Bantam.

18. Grijalbo, C. y Guzmán, J. F. (Noviembre de 2014). La inteligencia emocional del entrenador y la relación con su conducta verbal en competición. Conferencia llevada a cabo en el VIII Congreso Internacional de la Asociación Española de Ciencias del Deporte, Cáceres, Espańa.

19. Gutiérrez, L., Fontenla, E., Cons, M., Rodríguez, J. y Pazos, J. M. (2017). Mejora de la autoestima e inteligencia emocional a través de la psicomotricidad y de talleres de habilidades sociales. Sportis, 3(1), 187205.

20. Gutiérrez-Moret, M., Ibáńez-Martínez, R., Aguilar-Moya, R. y VidalInfer, A. (2016). Assessment of emotional intelligence in a simple of prospective secondary education teachers. Journal of Education for Teaching, 42(2), 123-134.

21. Hernández, A. y Palomera, R. (Marzo de 2004). Inteligencia emocional en el contexto educativo. Conferencia llevada a cabo en el IV Congreso de Psicología y Educación. Calidad Educativa (CD), Almería, Espańa.

22. Laborde, S., Dosseville, F. y Allen, M. S. (2016). Emotional intelligence in sport and exercise: A systematic review. Scandinavian Journal of Medicine \& Science in Sport, 26(8), 862-874

23. Lane, A. M. y Wilson, M. R. (2011). Emotions and trait emotional intelligence among ultra-endurance runners. Journal of Science and $\mathrm{Me}$ dicine in Sport, 14, 358-362.

24. Larramendi, E. (2015). La inteligencia emocional en el perfil del profesor de Educación Física de Primaria en Navarra (Trabajo fin de grado). Universidad Internacional de La Rioja, Logrońo, España.

25. López-Luque, V. (2017). Inteligencia emocional percibida y ansiedad en docentes (Trabajo fin de grado). Universidad de Granada, Granada, España.

26. Márquez-Cervantes, M. C. Y Gaeta-González, M. L. (2017). Desarrollo de competencias emocionales en pre-adolescentes: el papel de padres y docentes. Revista Electrónica Interuniversitaria de Formación del Profesorado, 20(2), 221-235.

27. Mayer, J. D. y Salovey, P. (1997). "What is emotional intelligence?" En P. Salovey y D. Sluyter (Eds.), Emotional development and emotional intelligence: Implications for educators (pp. 3-31). New York, Estados Unidos: Basic Books.

28. Méndez-Giménez, A., Fernández-Río, J. y Cecchini-Estrada, A. A (2013). Climas motivacionales, necesidades, motivación y resultados en Educación Física. Aula Abierta, 41(1), 63-72. 
29. Millán, A. C., García-Álvarez, D. J. y D’Aubeterre, M. E. (2014). Efecto de la inteligencia emocional y flujo de trabajo sobre estresores y bienestar psicológico: análisis de ruta en docentes. Revista Colombiana de Psicología, 23(1), 207-228.

30. Palomera, R., Fernández-Berrocal, P., \& Brackett, M. A. (2008). La inteligencia emocional como una competencia básica en la formación inicial de los docentes: algunas evidencias. Electronic journal of research in educational psychology, 6(2), 437-454.

31. Palomera, R., Fernández-Berrocal, P., y Brackett, M. A. (2008). La inteligencia emocional como una competencia básica en la formación inicial de los docentes: algunas evidencias. Electronic journal of research in educational psychology, 6(15), 437-454.

32. Palomera, R., Gil-Olarte, P. y Brackett, M.A. (2006). ¿Se perciben con inteligencia emocional los docentes? Posibles consecuencias sobre la calidad educativa. Revista de Educación, 341, 687-703.

33. Petrides, K. V., Sangareau, Y., Furnham, A. y Frederickson, N. (2006). Trait emotional intelligence and children's peer relations at school. Social Development, 15(3), 537-547.

34. Qualter, P., Whiteley, H. E., Hutchinson, J. M. y Pope, D. J. (2007). Supporting the development of emotional intelligence competencies to ease the transition from primary to high school. Educational psychology in practice, 23(1), 79-95.

35. Salovey, P. (2006). The Agenda for Future Research. En V. Druskat; F. Sala; G. Mount (Ed.), Linking EI and Performance at Work Current Research Evidence with Individuals and Groups (pp. 267-272).

36. Salovey, P., Mayer, J.D., Goldman, S.L., Turvey, C. y Palfai, T.P. (1995). Emotional attention, clarity and repair: exploring emotional intelligence using the Trair Meta-Mood Scale. En J.W. Pennebaker (Ed.), Emotion, disclosure, \& health (pp. 125-154). Washington DC, Estados Unidos: American Psychological Association.

37. Sánchez-Huéscar, I. (2018). Influencia de la inteligencia emocional en la competencia autopercibida de los docentes de Educación Infantil y Primaria de la Región de Murcia (Trabajo fin de grado). Universidad de Murcia, Murcia, España.

38. Troncoso, S. M., Burgos, C. J. y López-Walle, J. M. (2015). Climas motivacionales, liderazgo y cohesión grupal en contexto deportivo universitario. Educación Física y Ciencia, 17(1), 1-12.

39. Zizzi, S., Deaner, H. y Hirschhorn, D. (2003). The relationship between emotional intelligence and performance among college baseball players. Journal of Applied Sport Psychology, 15(3), 262-269. 
